\title{
Analysis of HST CMDs of 15 intermediate-age LMC clusters: self-consistent physical parameters and 3D distribution
}

\author{
L. O. Kerber ${ }^{1}$, B. X. Santiago ${ }^{2}$ and E. Brocato ${ }^{3}$ \\ ${ }^{1}$ IAG/USP, São Paulo , Brazil \\ email: kerber@astro.iag.usp.br \\ ${ }^{2}$ IF/UFRGS, Porto Alegre, Brazil \\ ${ }^{3}$ INAF-OAC, Teramo, Italy
}

\begin{abstract}
We analysed HST/WFPC2 colour-magnitude diagrams (CMDs) from 15 populous Large Magellanic Cloud (LMC) stellar clusters with ages between $\sim 0.3 \mathrm{Gyr}$ and $\sim 3$ Gyr. These $(\mathrm{V}, \mathrm{B}-\mathrm{V}) \mathrm{CMDs}$ are photometrically homogeneous and reach typically $\mathrm{V} \sim 22$. Accurate and selfconsistent physical parameters (age, metallicity, distance modulus and reddening) were extracted for each cluster by comparing the observed CMDs with synthetic ones. These determinations were made by means of simultaneous statistical comparison of MS fiducial lines and red clump position, offering objective and robust criteria to determine the best models. In general, the best models show a satisfactory fit to the data, adequately constraining the physical parameters of each cluster. The inferred spatial distribution of these clusters is roughly aligned with the LMC disk. The set of ages and metallicities homogeneously derived here can be used to calibrate integrated light studies applied to distant galaxies.
\end{abstract}

Keywords. galaxies: star clusters, Magellanic Clouds, Hertzsprung-Russell diagram

The LMC is a unique nearby case of a gas rich, star forming, irregular galaxy containing thousands of clusters with varying masses, ages and metallicities (Olszweski et al. 1996). Therefore, this cluster system is a useful record of the history of star formation, chemical enrichment and dynamics of a quite distinct type of galaxy relative to the Galaxy, being a key-piece to the studies in stellar populations. Although global features of this cluster system, like age and metallicity distributions and the age-metallicity relation, are relatively known (Olszewski et al. 1991, Geisler et al. 1997), the physical parameters of each cluster (specially age and distance) can be significantly better determined using sophisticated analysis of CMDs obtained with HST data, capable to resolve stars even in the cluster centre.

In the present work (Kerber et al. 2007) we analysed HST/WFPC2 CMDs from 15 populous LMC clusters (NGC 1651, 1718, 1777, 1831, 1856, 1868, 2121, 2155, 2162, 2173, 2209, 2213, 2249, SL506, 663) to determine the following physical parameters for each of them: age $(\tau)$, metallicity $(Z)$, distance modulus $\left((m-M)_{0}\right)$ and reddening $(E(B-V))$. For each cluster, the observed MS fiducial line and red clump (RC) position were simultaneously and statistically compared with the ones obtained from synthetic CMDs. The CMD models explored a regular grid in the parameter space consistent with previous determinations found in the literature. Control experiments were used to test our approach and to quantify formal uncertainties. Therefore, our determinations, based on photometrically homogeneous data (Brocato et al. 2001), are self-consistent and done by an objective and robust method. Figure 1, left panel, shows an example of data vs. 

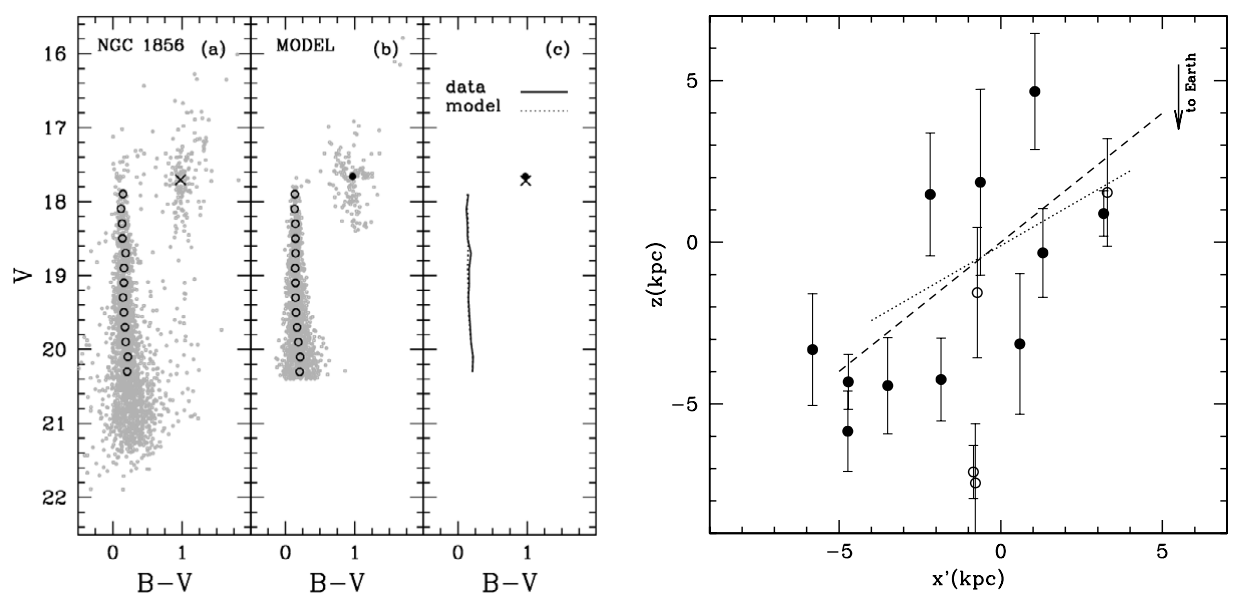

Figure 1. Left: data (panel a) vs. model (panel b) comparison for NGC 1856. Panel c confronts the fiducial lines and RC positions traced by the points in the previous panels. Right: spatial distribution of the clusters. The dotted line indicates the projected LMC disk as determined by Nikolaev et al. (2004). The linear fit for the clusters with the most reliable solutions (solid circles) is shown by the dashed line.

model comparison, where the agreement between the observed CMD of NGC 1856 and one of the best models for this cluster is very close.

In general, our results show a satisfactory fit to the data, reproducing well the MS fiducial line and RC features. Also, these models provide useful constraints to the physical parameters of each cluster, with typical uncertainties of 0.05 in $\log (\tau / \mathrm{yr}), 0.10 \mathrm{dex}$ in $[\mathrm{Fe} / \mathrm{H}], 0.05$ in $(m-M)_{0}$ and 0.02 in $E(B-V)$. We also recovered a mean $[\mathrm{Fe} / \mathrm{H}]$ of -0.50 for these intermediate-age clusters (IAC) in the LMC, in good agreement with the recent result based on spectroscopy of red giants (Grocholski et al. 2006).

Since we determined the individual distances for each cluster, we could probe the three-dimensional distribution for these IACs. As shown in the right panel of Fig. 1, if only the most reliable distance determinations are considered, this distribution seems to be roughly aligned with the LMC disk geometry, with a small difference of $\sim 8^{\circ}$ in the inclination. The alignment between the LMC disk and the IACs was also revealed by Grocholski et al. (this conference), with a good agreement in the individual distances for the common clusters in our samples. This not only adds reliability to the physical parameters derived by us in this work but also attests the success of this kind of approach in analysing CMDs of LMC clusters.

\section{Acknowledgements}

L.O.K. acknowledges FAPESP and IAU for the financial support to attend the IAUS241.

\section{References}

Brocato, E., Di Carlo, E. \& Menna, G. 2001, A\&A, 374, 523

Geisler, D., Bica, E., Dottori, H., et al. 1997, AJ, 114, 1920

Grocholski, A.J., Cole, A.A., Sarajedini, A., Geisler, D. \& Smith, V.V. 2006, AJ, 132, 1630

Kerber, L., Santiago, B. \& Brocato, E., 2007, A\&A, 462, 139

Nikolaev, S., Drake, A.J., Keller, S.C., et al. 2004, ApJ, 601, 260

Olszewski, E.W., Schommer, R.A., Suntzeff, N.B. \& Harris, H. 1991, AJ, 101, 515

Olszewski, E.W., Suntzeff, N.B. \& Mateo, M. 1996, ARA\&A, 34, 511 